\title{
THERMODYNAMICS OF VORTEX LINES IN LAYERED SUPERCONDUCTORS
}

\author{
Roberto Iengo and Claudio A. Scrucca \\ International School for Advanced Studies, Via Beirut 4, 34014 Trieste (Italy) \\ and INFN - Sezione di Trieste (Italy)
}

(February 1, 2008)

\begin{abstract}
We study the dissipative thermodynamics of vortex lines in layered superconductors within a simple string model in the dilute limit of negligible vortex interactions and compute the specific heat $C_{v}$ in presence of arbitrary dissipation. The interplay of dissipation, inertia and elasticity is shown to control the qualitative thermodynamical behavior and their relative amount determines two very distinct regimes for the specific heat. In the dissipation dominated case we find a behavior $C_{v} \sim \sqrt{T}$ for a large interval of temperature below $T_{c}$.
\end{abstract}

PACS: 74.60.Ge, 74.25.Bt. $\quad$ SISSA REF 125/97/EP

\section{INTRODUCTION}

As well known, type-II superconductors in a magnetic field exhibit an intermediate mixed phase characterized by vortex condensation and partial magnetic flux penetration through them. The dynamics of the latter crucially drives the electromagnetic behavior, and has been extensively studied in the last yearst. It has been shown that under appropriate assumptions, in particular for small magnetic field and vortex density, the elementary vortex excitations in thin layered samples with transverse magnetic field are flux lines crossing each layer only once, joining pancake vortices forming in each layer and moving in the xy plane. A well established and studied continuum modelit exist for these string-like excitations and it has been succesfully applied to analyze both the dynamical 2 and therodynamical ${ }^{5}$ - 6 behavior.

In the following we study the specific heat of vortex lines within a simple model which can be treated exactly and captures all the essential features of vortex dynamics relevant in layered superconductors. Dispersive and magnetic effects will be neglected, whereas dissipation $(\eta)$, inertia $(\mu)$ and elasticity $(\epsilon)$ will be taken into account exactly in the general case of a finite number $N$ of layers. We shall call $d$ the inter-layer distance, $N$ the number of layers, $L$ the thickness of the film and $A$ its area. The continuum limit $N \rightarrow+\infty$ will be analyzed as a particular limit in which the usual model holds. The problem has already geen addressed in the latter context by Blatter and Ivlevs for small magnetic fields and by Bulaevskii and Maley ${ }^{6}$ for large magnetic fields.

One of the central issues of this paper is to present a peculiar and unambiguous relation between the value of the ratio $I=\frac{\sqrt{\epsilon \mu}}{\eta d}$ and the thermodynamical properties of the vortex line. We show that there is a temperature $T_{o}=\frac{\pi}{2} \frac{\hbar}{k_{B}} \frac{\epsilon}{\eta L^{2}}$, typically of the order of a few $m K$, which separates the universal $I$-independent low temperature already discussed by Blatter and Ivlevt from an $I$-dependent higher temperature (but still below the critical temperature) behavior. In the relevant overdamped case in which $I \ll 1$, we find that in this regime the specific heat is proportional to the square root of the temperature. This should hold up to the critical temperature, provided that the model is still appropriate. In Section IV we summarize our results; we compare them with some previous results appeared in the litterature and discuss their physical relevance. In section $\mathrm{V}$ we discuss the effect of including the Magnus force. We find essentially the same results as before, in particular the same behavior with temperature, and even the numerical values of the coefficient are not very different.

The fundamental characteristics of the vortex lines are quite simple in the approximation we are considering; in particular, the self-energy stemming from the interaction between neighbor pancake vortices essentially reduces to a local elastic term, and dissipation seems to be reasonably well described by an ohmic viscous term. Both the elastic modulus $\epsilon$ and the friction coefficient $\eta$ can be theoretically computed, but the mass density $\mu$ is still object of discussion and can be only hardly estimated because of the wide class of contributions it gets depending on the particular setting, and its determination is still a central issue of vortex physics, even if there is strong belief for it to be negligibly small with respect to dissipation $\mathrm{B}$.

Since each point of the string has two-dimensional dynamics, we parameterize the problem in cylindrical coordinates, calling $\mathbf{q}(t, z)$ the xy position of the string at given $\mathrm{z}$. The equation of motion of the vortex line is

$$
\mu \ddot{\mathbf{q}}+\eta \dot{\mathbf{q}}-\epsilon \mathbf{q}^{\prime \prime}=0 .
$$

Because of the finite size of the system in the $z$ direction, it is necessary to impose the additional Neumann boundary conditions

$$
\mathbf{q}^{\prime}(t, 0)=\mathbf{q}^{\prime}(t, L)=0 .
$$

In this way, no energy-momentum flow is allowed across the end-points of the string.

The quantum-mechanical version of this dissipative dynamics is reached by means of Caldeira and Leggett's 
formalism 9 , in which dissipation is introduced by coupling the system to a thermal bath of harmonic oscillators with a continuous frequency spectrum satisfying the requirement to reproduce a simple velocity dependent ohmic viscous term in the effective equation of motion. The Euclidean effective action of the open system, relevant for thermodynamics, is obtained by integrating out the degrees of freedom of the bath, and reads in the continuum limit

$$
\begin{aligned}
S(\beta)= & \int_{0}^{L} d z \int_{0}^{\beta} d \tau\left[\frac{1}{2} \mu \dot{\mathbf{q}}^{2}(\tau, z)+\frac{1}{2} \epsilon \mathbf{q}^{\prime 2}(\tau, z)\right] \\
& +\frac{\eta}{4 \pi} \int_{0}^{L} d z \int_{0}^{\beta} d \tau \int_{0}^{\beta} d \tau^{\prime}\left[\frac{\mathbf{q}(\tau, z)-\mathbf{q}\left(\tau^{\prime}, z\right)}{\frac{\beta}{\pi} \sin \left(\pi \frac{\tau-\tau^{\prime}}{\beta}\right)}\right]^{2} .
\end{aligned}
$$

It is convenient to extend $\mathbf{q}(t, z)$ symmetrically to negative $z$, and expand the obtained even function in Fourier modes of fundamental frequency $\nu=\frac{\pi}{L}$ along $z$ by means of $\mathbf{q}(t, z)=\mathbf{q}_{0}(t)+\sqrt{2} \sum_{n=0}^{N-1} \mathbf{q}_{n}(t) \cos \nu n z ;$ in this way, the boundary conditions are automatically satisfied. The Euclidean action then splits into a sum of harmonic oscillator contributions encoding the vibrational modes of the string plus a zero mode representing the translational motion of the center of mass.

In order to handle the thermodynamical Euclidean path-integral, we introduce the Matsubara frequency $\omega=\frac{2 \pi}{\beta}$ and expand the periodic configurations $\mathbf{q}_{n}(\tau)$ involved in the path-integral for the partition function in Fourier modes with fundamental frequency $\omega$ along imaginary time, taking $\mathbf{q}_{n}(\tau)=\frac{1}{\sqrt{\beta}} \sum_{k=-\infty}^{+\infty} \mathbf{q}_{n k} e^{i \omega k \tau}$. The dissipative term, once Fourier-transformed, will contribute a peculiar $|\omega k|$ term, remnant of the derivative nature of ohmic dissipation.

In Fourier space, the effective action is

$$
S(\beta)=\frac{1}{2} \sum_{n=0}^{N-1} \sum_{k=-\infty}^{+\infty}\left[M\left(\omega_{k}^{2}+\Omega_{n}^{2}\right)+\Lambda\left|\omega_{k}\right|\right]\left|\mathbf{q}_{n k}\right|^{2} .
$$

$M=\mu L$ is the total mass, $\Lambda=\eta L$ the total friction coefficient and $\Omega=\sqrt{\frac{\epsilon}{\mu}} \frac{\pi}{L}$ the characteristic vibration frequency; we have used the notation $\omega_{k}=\omega k$ and $\Omega_{n}=$ $\Omega n$. Since there is only a finite number $N$ of layers, we have cut-off the possible modes at that value.

A more natural way back to the finite $N$ case is to write a discrete action in terms of the single pancake vortices coordinates $\mathbf{x}_{l}(t)=\mathbf{q}(t, l d)$. One then obtains the action for $N$ pancake vortices of mass $\mu d$ and friction coefficient $\eta d$, each harmonically coupled to its nearest neighbors with elastic modulus $\frac{\epsilon}{d}$. The decoupling of these degrees of freedom can be achieved performing a change of variable analogous to the Fourier expansion of the continuum case, defining $\mathbf{q}_{n}(t)$ from $\mathbf{x}_{l}(t)$ through $\mathbf{x}_{l}(t)=\mathbf{q}_{o}(t)+\sqrt{2} \sum_{n=1}^{N-1} \mathbf{q}_{n}(t) \cos \left(\nu n\left(l+\frac{1}{2}\right) d\right)$. One then obtains the action (4), but with a modified frequency spectrum given by

$$
\Omega_{n}=\frac{2 N}{\pi} \sin \left(\frac{\pi}{2} \frac{n}{N}\right) \Omega .
$$

Obviously, in the limit of $N \rightarrow+\infty$, the lattice version of the theory reduces to the continuum formulation, with the same frequency spectrum $\Omega_{n}=\Omega n$.

In the following, for the sake of clearness we will concentrate on the basic case of absence of pinning of vortex lines by impurities and defects, and focus on the effects of elasticity and dissipation (the effect of the Magnus force will instead be considered in Section V). However, the treatment of this important generalization could be easily faced in exactly the same way; introducing a single columnar pinning center through a harmonic confining potential with elastic modulus $k$ and characteristic frequency $\Omega_{p}=\sqrt{\frac{k}{m}}$, the only novel feature would be the modification of the spectrum of the modes to $\Omega_{n}^{*}=\sqrt{\Omega_{n}^{2}+\Omega_{p}^{2}}$.

\section{PARTITION FUNCTION}

We have seen that the dynamics of the vortex line is encoded in a zero mode describing its translational motion and $N-1$ harmonic modes with frequency spectrum $\Omega_{n}$ describing its vibration. Consequently, the partition function,

$$
\begin{aligned}
Z(\beta) & =\operatorname{Tr}^{-\beta H} \\
& =\int_{\mathbf{q}(0, z)=\mathbf{q}(\beta, z)} \mathcal{D} \mathbf{q}(\tau, z) e^{-S(\mathbf{q}(\tau, z)),}
\end{aligned}
$$

will factorize into the product of the partition functions of the modes. Thus, all that we need to know for our aim is the dissipative thermodynamics of the zero-mode degree of freedom and the remaining harmonic modes. The path-integral for the latter can be evaluated quite easily in both cases since it is Gaussian, and reduces to a product of ordinary integrals in Fourier space.

\section{A. Zero-mode}

For the zero mode, we get

$$
\begin{aligned}
Z(\beta, \Lambda) & =\mathcal{N}(\beta) \prod_{k=0}^{+\infty} \iint d \mathbf{q}_{o k} d \mathbf{q}_{o k}^{*} e^{-\mathbf{q}_{o k}^{*}\left(M \omega_{k}^{2}+\Lambda \omega_{k}\right)} \mathbf{q}_{o k} \\
& =\mathcal{N}^{\prime}(\beta) A \prod_{k=1}^{+\infty}\left(1+\frac{\gamma}{\omega} \frac{1}{k}\right)^{-2}
\end{aligned}
$$

where $\gamma=\frac{\Lambda}{M}=\frac{\eta}{\mu}$ is the characteristic frequency related to dissipation and $A$ is the area. The normalization factor is fixed by requiring that in the limit $\Lambda \rightarrow 0$ the partition function reduces to the free one $Z_{o}(\beta)=\frac{M}{2 \pi \beta} A$. It follows 


$$
\mathcal{N}^{\prime}(\beta)=\frac{M}{2 \pi \beta} .
$$

Unfortunately, the infinite product representing the effect of dissipation is divergent. We then have to introduce a frequency cut-off $\omega_{c}$ above which the effect of dissipation is assumed to be small, or at least not adequately described by a simple ohmic viscous term in the equation of motion, and truncate the product at $k_{c}(\omega)=\frac{\omega_{c}}{\omega}$. Using the infinite product representation of the $\Gamma$-function, Eq. (A2), we obtain for $k_{c}(\omega) \rightarrow+\infty$,

$$
\prod_{k=1}^{k_{c}(\omega)}\left(1+\frac{\gamma}{\omega} \frac{1}{k}\right)=\frac{k_{c}(\omega)^{\frac{\gamma}{\omega}}}{\frac{\gamma}{\omega} \Gamma\left(\frac{\gamma}{\omega}\right)} .
$$

The same result capbe obtained using the Drude model as regularization 10 1 11 , where the frequency spectrum of the thermal bath is cut-off at $\omega_{c}$ dividing it by a factor $1+\left(\frac{\omega}{\omega_{c}}\right)^{2}$; the cut-off frequency can then be related to a microscopic relaxation time $\tau_{c}$ by $\omega_{c}=\frac{2 \pi}{\tau_{c}}$.

The final form for the partition function is conveniently written in terms of the function

$$
\tilde{\Gamma}(z)=\frac{1}{\sqrt{2 \pi}} z^{\frac{1}{2}-z} e^{z} \Gamma(z),
$$

which goes to one for large arguments away from the real negative axis (see Eq. A5)); one gets

$$
Z(\beta, \gamma)=\frac{\Lambda}{2 \pi} A \tilde{\Gamma}^{2}\left(\frac{\gamma}{\omega}\right) e^{-\beta \mathcal{E}_{o}(\gamma)},
$$

with a zero-point energy

$$
\mathcal{E}_{o}(\gamma)=\frac{\gamma}{\pi}\left(1+\ln \frac{\omega_{c}}{\gamma}\right) .
$$

Quite interestingly, we learn that, since the partition function depends only on $\frac{\omega}{\gamma}$ apart from an irrelevant multiplicative constant and the zero-point energy, the effect of dissipation on the thermodynamics of the zero-mode is only to modify the temperature scale. This could have been expected from dimensional analysis, since the only energy scale we can construct from $M$ and $\Lambda$ is $\gamma$ (we do not consider $\omega_{c}$ since we expect dissipation to manifest in a universal ohmic way in the limit of small relaxation time $\tau_{c}$, situation in which the only role of $\omega_{c}$ should be to renormalize the energy of the open system), and the only dimensionless quantity that can enter the partition function is the reduced temperature $\frac{\omega}{\gamma}$.

Notice that in the limit of strong damping $\frac{\gamma}{\omega} \gg 1$, that is equivalent to the small mass limit $\mu \rightarrow 0$ since $\frac{\omega}{\gamma}=$ $\frac{\mu \omega}{\eta}$, we are left, apart from the zero-point energy, with a trivial constant partition function $Z_{l}(\beta, \gamma)$ independent of $\mu$; using Eq. A5

$$
\begin{aligned}
Z(\beta, \gamma) \underset{\frac{\gamma}{\omega} \gg 1}{\longrightarrow} & \frac{\Lambda}{2 \pi} A\left[1+\frac{\omega}{12 \gamma}\right]^{2} e^{-\beta \mathcal{E}_{o}(\gamma)} \\
\simeq & \frac{\Lambda}{2 \pi} A e^{-\beta \mathcal{E}_{o}(\gamma)} .
\end{aligned}
$$

\section{B. Harmonic modes}

For the other modes, we proceed exactly in the same way and the partition function is given by

$$
\begin{aligned}
& Z\left(\beta, \Omega_{n}, \Lambda\right)= \\
& \quad=\mathcal{N}(\beta) \prod_{k=0}^{+\infty} \iint d \mathbf{q}_{n k} d \mathbf{q}_{n k}^{*} e^{-\mathbf{q}_{n k}^{*}\left[M\left(\omega_{k}^{2}+\Omega_{n}^{2}\right)+\Lambda \omega_{k}\right] \mathbf{q}_{n k}} \\
& \quad=\mathcal{N}^{\prime}(\beta) \frac{1}{\Omega_{n}^{2}} \prod_{k=1}^{+\infty}\left[1+\frac{\gamma}{\omega} \frac{1}{k}+\left(\frac{\Omega_{n}}{\omega}\right)^{2} \frac{1}{k^{2}}\right]^{-2}
\end{aligned}
$$

The requirement this to reduce to the pure harmonic oscillator result $Z_{o}\left(\beta, \Omega_{n}\right)=\left(2 \sinh \frac{\beta \Omega_{n}}{2}\right)^{-2}$ in the limit $\Lambda \rightarrow 0$ fixes, using Eq. (A1), the normalization coefficient to

$$
\mathcal{N}^{\prime}(\beta)=\frac{1}{\beta^{2}} .
$$

As before, the dissipative term is responsible for a divergence in the correction factor accounting for the damping of the system; introducing the same frequency cut-off, the product can be carried out by factorization, obtaining

$$
\begin{aligned}
\prod_{k=1}^{k_{c}(\omega)}[1 & \left.+\frac{\gamma}{\omega} \frac{1}{k}+\left(\frac{\Omega_{n}}{\omega}\right)^{2} \frac{1}{k^{2}}\right]= \\
& =\frac{k_{c}(\omega) \frac{\gamma}{\omega}}{\left(\frac{\Omega_{n}}{\omega}\right)^{2} \Gamma\left(\frac{\gamma}{\frac{\gamma}{\omega}+i \xi_{n}} \frac{\gamma}{\omega}\right) \Gamma\left(\frac{\frac{\gamma}{2}-i \xi_{n}}{\omega}\right)},
\end{aligned}
$$

where $\xi_{n}=\sqrt{\Omega_{n}^{2}-\frac{\gamma^{2}}{4}}$. Again, the same result can be obtained using the Drude model as regularization $1 \mathrm{~d}, \mathrm{~L} 1 \mathrm{1}$, allowing a microscopic interpretation of the cut-off.

The partition function can then be written, using again the $\tilde{\Gamma}$-function defined above, as

$$
Z\left(\beta, \Omega_{n}, \gamma\right)=\tilde{\Gamma}^{2}\left(\frac{\frac{\gamma}{2}+i \xi_{n}}{\omega}\right) \tilde{\Gamma}^{2}\left(\frac{\frac{\gamma}{2}-i \xi_{n}}{\omega}\right) e^{-\beta \mathcal{E}_{o}\left(\Omega_{n}, \gamma\right)},
$$

with a zero-point energy given by

$$
\mathcal{E}_{o}\left(\Omega_{n}, \gamma\right)=\frac{\gamma}{\pi}\left(1+\ln \frac{\omega_{c}}{\Omega_{n}}\right)-i \frac{\xi_{n}}{\pi} \ln \frac{\frac{\gamma}{2}+i \xi_{n}}{\frac{\gamma}{2}-i \xi_{n}} .
$$

In the strongly dissipated regime, which means $\frac{\gamma}{\Omega} \gg n$, and successively in the limit of low temperature with respect to damping, corresponding to $\frac{\gamma}{\omega} \gg 1$, the partition function is seen, using respectively Eqs. (A4) and (A5), to reduce to

$$
\begin{aligned}
Z\left(\beta, \Omega_{n}, \gamma\right) \underset{\frac{\gamma}{2} \gg n}{\longrightarrow} & \tilde{\Gamma}^{2}\left(\frac{\gamma}{\omega}\right) \tilde{\Gamma}^{2}\left(\frac{\Omega_{n}^{2}}{\gamma \omega}\right) e^{-\beta \mathcal{E}_{o}(\gamma)} \\
\underset{\frac{\gamma}{\omega} \gg 1}{\longrightarrow} & {\left[1+\frac{\omega}{12 \gamma}\right]^{2} \tilde{\Gamma}^{2}\left(\frac{\Omega_{n}^{2}}{\gamma \omega}\right) e^{-\beta \mathcal{E}_{o}(\gamma)} } \\
& \simeq \tilde{\Gamma}^{2}\left(\frac{\Omega_{n}^{2}}{\gamma \omega}\right) e^{-\beta \mathcal{E}_{o}(\gamma)}
\end{aligned}
$$


The last extreme limit can be seen as a small mass limit $\mu \rightarrow 0$. Since the variable $\frac{\gamma \omega}{\Omega^{2}}=\left(\frac{N}{\pi}\right)^{2} \frac{\eta d^{2}}{\epsilon} \omega$ is independent of $\mu$, the resulting behavior $Z_{l}\left(\beta, \Omega_{n}, \gamma\right)$ no longer depends on $\mu$ apart from the zero-point contribution $\mathcal{E}_{o}(\gamma)$, which is found to be the same as the one for the zero-mode, Eq. (12). Again, this is obvious from dimensional analysis. The only dimensionless quantity independent of $\mu$ we can form from the three variables $\omega, \gamma$ and $\Omega$ is $\frac{\gamma \omega}{\Omega^{2}}$, indeed entering the resulting behavior; instead, the $\mu$-dependent variable $\frac{\omega}{\gamma}=\frac{\mu \omega}{\eta}$ disappears in the limit. Having eliminated one of the scales, we fall in a regime in which, as for the zero-mode, dissipation manifests itself only in the temperature scale.

\section{Vortex-string}

In order to compute the partition function of the string, all that we have to do is multiply those relative to the modes that we have just computed, that is

$$
\begin{aligned}
Z_{v o r}(\beta, \Omega, \gamma)=Z(\beta, \gamma) & \prod_{n=1}^{N-1} Z\left(\beta, \Omega_{n}, \gamma\right) \\
= & \frac{\Lambda}{2 \pi} A \tilde{\Gamma}^{2}\left(\frac{\gamma}{\omega}\right) \prod_{n=1}^{N-1} \tilde{\Gamma}^{2}\left(\frac{\frac{\gamma}{2}+i \xi_{n}}{\omega}\right) \tilde{\Gamma}^{2}\left(\frac{\frac{\gamma}{2}-i \xi_{n}}{\omega}\right) \\
& e^{-\beta E_{o}(\Omega, \gamma)}
\end{aligned}
$$

The zero-point energy is the sum of those of the modes

$$
E_{o}(\Omega, \gamma)=\mathcal{E}_{o}(\gamma)+\sum_{n=1}^{N-1} \mathcal{E}_{o}\left(\Omega_{n}, \gamma\right)
$$

The first observation we can do is that for fixed $\omega$, $\gamma$ and $\Omega$, the factor $Z\left(\beta, \Omega_{n}, \gamma\right)$ in the product over the modes in the partition function goes to one for $n \rightarrow+\infty$, and the latter is thus a well defined function of these variable for any $N$, since the product can be shown to converge. The meaning of this observation is that the variables we have chosen represent the true physical scales of the problem, and increasing $N$ just add higher frequency modes that are more and more suppressed on the fixed scale we consider.

Conversely, the zero-point energy increases indefinitely with $N$. Using the lattice regularization and the results derived in appendix B, the total zero-point energy can be recast in the following form

$$
E_{o}(\Omega, \gamma)=E_{\text {odiv }}(\Omega, \gamma)+E_{\text {ofin }}(\Omega, \gamma),
$$

with

$$
\begin{aligned}
E_{\text {odiv }}(\Omega, \gamma)= & \left\{\frac{N}{\pi}\left(\frac{4 N}{\pi}-1\right) \Omega-\frac{1}{8} \ln N \frac{\gamma^{2}}{\Omega}\right. \\
& \left.+\left[\frac{N}{\pi} \ln \left(\frac{\pi}{N} \frac{\omega_{c}}{\Omega}\right)+\frac{\ln N}{2 \pi}\right] \gamma\right\},
\end{aligned}
$$

$$
\begin{aligned}
E_{o f i n}(\Omega, \gamma)= \\
=-\left\{\frac{1}{12} \Omega-\frac{1}{\pi}\left[1-\ln \left(\pi \frac{\gamma}{\Omega}\right)\right] \gamma+\frac{1}{8} \ln G \frac{\gamma^{2}}{\Omega}\right\} \\
-\sum_{n=1}^{N-1}\left[\Omega_{n}-\frac{\gamma}{\pi}-\frac{1}{8} \frac{\gamma^{2}}{\Omega_{n}}+i \frac{\xi_{n}}{\pi} \ln \frac{\frac{\gamma}{2}+i \xi_{n}}{\frac{\gamma}{2}-i \xi_{n}}\right] .
\end{aligned}
$$

The last sum is finite also for $N \rightarrow+\infty$. Notice also that it vanishes in the free case $\gamma \rightarrow 0$.

In the limit in which each of the $N$ modes is in the strong damping regime and at low temperatures with respect to friction, meaning $\frac{\gamma}{\Omega} \gg N$ and $\frac{\gamma}{\omega} \gg 1$, and corresponding to a limit of vanishing mass $\mu \rightarrow 0$, the partition function first factorizes essentially into the product of two functions depending respectively on the combinations $\frac{\omega}{\gamma}=\frac{\mu \omega}{\eta}$ and $\frac{\gamma \omega}{\Omega^{2}}=\left(\frac{N}{\pi}\right)^{2} \frac{\eta d^{2}}{\epsilon} \omega$, and then simplifies to a function $Z_{l v o r}(\beta, \Omega, \gamma)$ which, apart from the zeropoint energy, depends only on the $\mu$-independent variable $\frac{\gamma \omega}{\Omega^{2}}$. In fact,

$$
\begin{aligned}
& Z_{v o r}(\beta, \Omega, \gamma) \rightarrow \\
& \underset{\frac{\gamma}{\Omega} \gg N}{\longrightarrow} \frac{\Lambda}{2 \pi} A \tilde{\Gamma}^{2 N}\left(\frac{\gamma}{\omega}\right) \prod_{n=1}^{N-1} \tilde{\Gamma}^{2}\left(\frac{\Omega_{n}^{2}}{\gamma \omega}\right) e^{-\beta E_{o}(\gamma)} \\
& \underset{\frac{\gamma}{\omega} \gg 1}{\longrightarrow} \frac{\Lambda}{2 \pi} A\left[1+\frac{\omega}{12 \gamma}\right]^{2 N} \prod_{n=1}^{N-1} \tilde{\Gamma}^{2}\left(\frac{\Omega_{n}^{2}}{\gamma \omega}\right) e^{-\beta E_{o}(\gamma)} \\
& \simeq \frac{\Lambda}{2 \pi} A \prod_{n=1}^{N-1} \tilde{\Gamma}^{2}\left(\frac{\Omega_{n}^{2}}{\gamma \omega}\right) e^{-\beta E_{o}(\gamma)}
\end{aligned}
$$

where now the zero-point energy has simplified to

$$
E_{o}(\gamma)=N \mathcal{E}_{o}(\gamma)=N \frac{\gamma}{\pi}\left(1+\ln \frac{\omega_{c}}{\gamma}\right) .
$$

Again, we see that the product in the partition function is well defined for any $N$ since the $n$-th factor goes quickly to one for $n \rightarrow+\infty$; the zero-point energy grows indefinitely if one add modes increasing $N$, as in the general case.

In the following, we shall distinguish between two regimes in the thermodynamics of the vortex line: the underdamped one, for which $\frac{\gamma}{\Omega} \ll N$, and the overdamped one, for which $\frac{\gamma}{\Omega} \gg N$.

\section{SPECIFIC HEAT}

Having computed the partition function of the vortex line, we can easily compute its specific heat. The latter can be defined in presence of dissipation as the derivative of the mean energy of the open system with respect to temperature, considering dissipation just as an additional mechanism for exchanging energy with the thermal bath and modifying the response of the system under heating.

In fact, within the Caldeira-Leggett formulation, the partition function of the complete system factorizes into 
the partition function of the bath and that of the open system with an effective action containing the influence of dissipation. Technically, this is implemented with an appropriate shift in the bath's oscillators coordinates which are just dummy variables in the path-integral 10 . Thus, in this scheme, the total heat capacity of the system is the sum of the heat capacity of the bath alone plus the one of the vortex lines.

One of the interesting features of the specific heat is that it is independent of the rather unknown parameter $\omega_{c}$ entering the zero-point energy. Also, from our previous discussion, we expect it to remain well behaved in the $\mu \rightarrow 0$ limit.

\section{A. General expression}

The specific heat of a single vortex line is

$$
C_{v o r}(\beta, \Omega, \gamma)=-\frac{\partial^{2}}{\partial T \partial \beta} \ln Z_{s t r}(\beta, \Omega, \gamma) .
$$

From now on, we will work with the reduced specific heat, dropping a factor $k_{B}$. The usual one is obtained by multiplying the latter by $k_{B}$ and by the density of vortices in the sample.

The result for the specific heat is easily computed as a sum over the contributions of the modes and is found to be

$$
\begin{aligned}
C_{v o r}(\beta, \Omega, \gamma)=C(\beta, \gamma)+\sum_{n=1}^{N-1} C\left(\beta, \Omega_{n}, \gamma\right) \\
=2\left(\frac{\gamma}{\omega}\right)^{2} \tilde{\Psi}^{\prime}\left(\frac{\gamma}{\omega}\right) \\
+2 \sum_{n=1}^{N-1}\left\{\left(\frac{\frac{\gamma}{2}+i \xi_{n}}{\omega}\right)^{2} \tilde{\Psi}^{\prime}\left(\frac{\frac{\gamma}{2}+i \xi_{n}}{\omega}\right)\right. \\
\left.+\left(\frac{\frac{\gamma}{2}-i \xi_{n}}{\omega}\right)^{2} \tilde{\Psi}^{\prime}\left(\frac{\frac{\gamma}{2}-i \xi_{n}}{\omega}\right)\right\},
\end{aligned}
$$

where

$$
\tilde{\Psi}^{\prime}(z)=\frac{d^{2}}{d z^{2}} \ln \tilde{\Gamma}(z)
$$

and goes to zero for large arguments away from the real negative axis, Eq. A6. At high temperature, the specific heat saturates at the value $2 N-1$; all the $N-1$ harmonic modes contributes 2 , whereas the zero-mode only 1, as seen from Eq. (A8).

In the limit of strong damping for each of the $N$ modes, $\frac{\gamma}{\Omega} \gg N$, we have $\frac{\frac{\gamma}{2} \pm i \xi_{n}}{\omega} \rightarrow \frac{\Omega_{n}^{2}}{\gamma \omega}, \frac{\gamma}{\omega}$. At low temperatures with respect to friction, $\frac{\gamma}{\omega} \gg 1$, the specific heat simplifies to

$$
C_{v o r}(\beta, \Omega, \gamma) \rightarrow
$$

$$
\begin{gathered}
\underset{\frac{\gamma}{\Omega} \gg N}{\longrightarrow} 2 N\left(\frac{\gamma}{\omega}\right)^{2} \tilde{\Psi}^{\prime}\left(\frac{\gamma}{\omega}\right)+2 \sum_{n=1}^{N-1}\left(\frac{\Omega_{n}^{2}}{\gamma \omega}\right)^{2} \tilde{\Psi}^{\prime}\left(\frac{\Omega_{n}^{2}}{\gamma \omega}\right) \\
\underset{\frac{\gamma}{\omega} \gg 1}{\longrightarrow} \frac{N}{3} \frac{\omega}{\gamma}+2 \sum_{n=1}^{N-1}\left(\frac{\Omega_{n}^{2}}{\gamma \omega}\right)^{2} \tilde{\Psi}^{\prime}\left(\frac{\Omega_{n}^{2}}{\gamma \omega}\right) \\
\simeq 2 \sum_{n=1}^{N-1}\left(\frac{\Omega_{n}^{2}}{\gamma \omega}\right)^{2} \tilde{\Psi}^{\prime}\left(\frac{\Omega_{n}^{2}}{\gamma \omega}\right) .
\end{gathered}
$$

In the final extreme limit, the specific heat reduces to a function $C_{\text {lvor }}(\beta, \Omega, \gamma)$ which depends only on the $\mu$ independent variable $\frac{\gamma \omega}{\Omega^{2}}$.

\section{B. Behavior in various regimes}

As shown, the variables which naturally enter the thermodynamics of the string have the dimensions of an energy and are the Matsubara frequency $\omega=2 \pi k_{B} T$ which is proportional to the thermal energy, the characteristic frequency $\Omega=\sqrt{\frac{\epsilon}{\mu}} \frac{\pi}{L}$ of the string and $\gamma=\frac{\eta}{\mu}$ which is the characteristic energy of dissipative processes. These variables represent the dependence on temperature and damping on a physical energy scale that depends on $L=N d$ and thus on $N$, the number of layers.

It is convenient to define the reduced temperature $t$ and the dimensionless damping parameter $\alpha$ according to

$$
\begin{aligned}
& t=\frac{\omega}{\Omega}=2 N \sqrt{\frac{\mu}{\epsilon}} d k_{B} T=\frac{\pi I}{N} \frac{T}{T_{o}}, \\
& \alpha=\frac{\gamma}{\Omega}=\frac{N}{\pi} \frac{\eta d}{\sqrt{\epsilon \mu}}=\frac{N}{\pi I} .
\end{aligned}
$$

The $\mu$-independent variable arizing in the $\mu \rightarrow 0$ limit is then simply

$$
\alpha t=\frac{\omega \gamma}{\Omega^{2}}=\frac{2 N^{2}}{\pi} \frac{\eta d^{2}}{\epsilon} k_{B} T=\frac{T}{T_{o}} .
$$

The meaning of a possible continuum limit $N \rightarrow+\infty$ can now be better elucidated. In order to compare situations with different $N$ s, we work with the $N$-dependent variables $\alpha$ and $t$, so that adding modes does not change the contributions of those that were already present but constitute only a correction. The continuum limit will then be relevant as a reliable approximation to the finite $N$ case whenever the modes with $n>N$ give a negligible contribution to thermodynamics. As we will see, this will be true for low temperatures.

As we already pointed out, the thermodynamics of the vortex line clearly exhibits two distinct and very different regimes. We shall call the underdamped regime the case in which $\alpha \ll N$, and the overdamped regime the case in which $\alpha \gg N$, and analyze them separately.

The global behavior of the specific heat is shown in Fig. 1. There are two regimes according to the value of $\alpha$. For 
$\alpha \ll N$, the underdamped case, the specific heat depends only weakly on it and the effect of increasing $N$ is to raise the temperature at which the saturation to the asymptotic value $2 N-1$ begins. For $\alpha \gg N$, the overdamped case, the specific heat acquires a strong dependence on $\alpha$. Increasing the temperature, the specific heat raises very quickly to the value $N-1$, going approximately like a square root, and continues to grow very slowly and almost linearly towards its high temperature saturation value $2 N-1$.

In the limit of very small mass, the specific heat stop its raising at $N-1$ instead of $2 N-1$. This is due to the fact that the extreme damping has killed almost all the kinetic part of dynamics, corresponding to an asymptotic specific heat equal to 0 instead of 1 for the center of mass particle degree of freedom (which gets completely killed) and equal to 1 instead of 2 for each of the harmonic modes (for which the kinetic and potential part of the energy are equal in the free case). Moreover, if damping is strong enough with respect to inertia, the specific heat depends only on the $\mu$-independent variable $\alpha$.

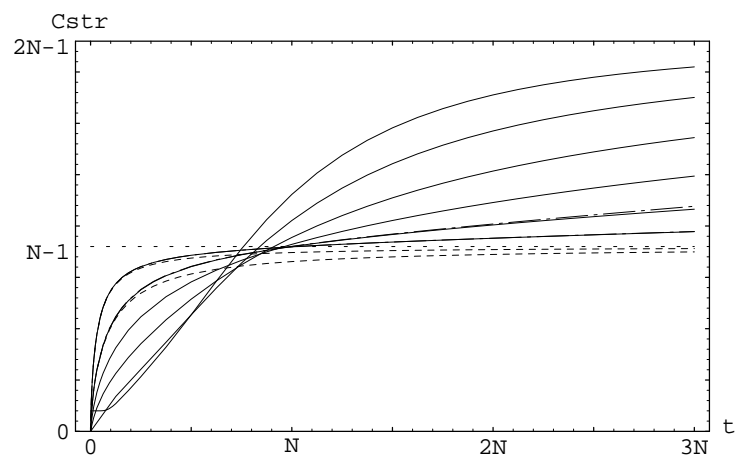

FIG. 1. The specific heat $C_{\text {vor }}(t, \alpha)$ of the vortex-string as a function of the reduced temperature $t$. The solid lines refers to $\alpha=0,3, N, 2 N, 4.5 N, 12 N$. For $\alpha=4.5 N, 12 N$, the limiting strong damping scaling behavior with (dot-dashed lines) and without (dashed lines) the linear correction (term $\frac{N}{3} \frac{\omega}{\gamma}$ in Eq.(30)) is seen to be an increasingly good approximation.

\section{Underdamped regime: $\alpha \ll N(I \gg 1)$}

In this regime, we use Eq. (28), which depend on both $\alpha$ and $t$. Notice first that in the high temperature limit, $t \gg N$, the specific heat tends to its maxmimal value $2 N-1$ and is almost constant. Next consider not too high temperatures, $t \ll N$. The contribution of the modes with $n>N$ in the sum giving the total specific heat would give a negligible contribution (since the arguments of both the $\tilde{\Psi}$-functions would be large), and the continuum limit is a good approximation. We can thus take the limit $N \rightarrow+\infty$ keeping $\alpha$ and $t$ fixed. In this way, the asymptotic behaviors of the specific heat in the extremes of this range of temperature can be computed and finally

$$
C_{v o r}(t, \alpha) \simeq\left\{\begin{array}{l}
\frac{1}{3}\left(\frac{1}{\alpha}+\frac{\pi^{2}}{6} \alpha\right) t, t \ll 1 \\
\frac{\pi}{3} t, 1 \ll t \ll N \\
2 N-1, t \gg N
\end{array} .\right.
$$

The behavior for $t \ll 1$ directly follows from the asymptotic behavior of the $\tilde{\Psi}^{\prime}$ - function, Eq. (A6), whereas the one for $1 \ll t \ll N$ is obtained approximating the sum in the specific heat with an integral.

At low temperatures, $t \ll N$, the underdamped case further splits into three sub-regimes. For values of $\alpha$ below a first critical value $\alpha_{c 1} \simeq 0.4$, the specific heat progressively pass from the free shape to a linearly growing one; moreover, any infinitesimal amount of dissipation, $\alpha>0$, causes the specific heat to start from zero instead of one, because of the center of mass contribution. For $\alpha$ beyond a second critical value $\alpha_{c 2} \simeq 1.5$, the specific heat starts to deviate from the linear shape, getting more and more convex at low temperature. Finally, for $\alpha$ between the two critical values, there is no substantial dependence of the specific heat on friction, and the response to heating is approximately linear in the temperature in the whole continuum region $t \ll N$.

The two critical values of $\alpha$ can be obtained by requiring the matching of the behaviors for $t \ll 1$ and $1 \ll t \ll N$. They satisfy thus the quadratic equation

$$
\frac{1}{3}\left(\frac{1}{\alpha_{c}}+\frac{\pi^{2}}{6} \alpha_{c}\right)=\frac{\pi}{3}
$$

which yields

$$
\alpha_{c 1,2}=\frac{3 \mp \sqrt{3}}{\pi},
$$

in agreement with the values extracted from the plot.

\section{Overdamped regime: $\alpha \gg N(I \ll 1)$}

In this regime, we can use Eq. (30), which depend only on $\alpha t=\frac{T}{T_{o}}$. At high temperature, $\alpha t \gg N^{2}$, the specific heat grows linearly from the value $N-1$ with a slope $\frac{N}{3 \alpha}$ until $\alpha t \sim \alpha^{2}$, where it starts saturating to its asymptotic value $2 N-1$. For low temperature limit, $\alpha t \ll N^{2}$, the contribution of the modes with $n>N$ in the sum giving the total specific heat are again negligible, and we can take the continuum limit $N \rightarrow+\infty$ keeping $\alpha t$ fixed. In this case, the asymptotic behaviors of the specific heat at the extremes of the low temperature region can be found and finally the behavior is the following 


$$
C_{\text {vor }}(t, \alpha) \simeq\left\{\begin{array}{l}
\frac{\pi^{2}}{18} \alpha t, \alpha t \ll 1 \\
2 C_{o} \sqrt{\alpha t}, 1 \ll \alpha t \ll N^{2} \\
N-1+\frac{N}{3 \alpha} t, N^{2} \ll \alpha t \ll \alpha^{2}=\frac{N^{2}}{\pi^{2} I^{2}} \\
2 N-1, \alpha t \gg \alpha^{2}=\frac{N^{2}}{\pi^{2} I^{2}}
\end{array},\right.
$$

where $C_{o}=0.490$ is the constant given by the integral (A11) quoted in appendix A. As before, the behavior for $\alpha t \ll 1$ directly stems from the asymptotic behavior of the $\tilde{\Psi}^{\prime}$-function, Eq. (A6), whereas the one for $1 \ll \alpha t \ll$ $N^{2}$ is computed approximating the sum over the modes with an integral.

The reduced temperatures that are important for the shape transitions in the specific heat are recognized to be $t=1, N$ in the underdamped case and $t=\frac{1}{\alpha}, \frac{N^{2}}{\alpha}, \alpha$ in the overdamped one. The important question that we shall now address is whether some of these can be relevant at the superconductivity temperature scale.

\section{DISCUSSION}

The available experimental and theoretical work on superconductors and vortex dynamics does not allow for a precise knowledge of all the parameters entering the description of the problem. In particular, whereas the damping and elasticity coefficients can be computed, the mass density is ambiguous. We will thus focus on $I=\frac{\sqrt{\epsilon \mu}}{\eta d}$ as the fundamental unknown quantity carrying the dependence on $\mu$.

The value of $\eta$ can be estimated at low temperaure using the Bardeen-Stephen expression 12 , whereas $\epsilon$ is known from the microscopic theory; in Gaussian units (and recovering $\hbar$ ),

$$
\eta=\frac{\Phi_{o}^{2}}{2 \pi c^{2} \xi^{2} \rho_{N}}, \epsilon=\kappa^{2}\left(\frac{\Phi_{o}}{4 \pi \lambda}\right)^{2} \ln \frac{\lambda}{\kappa \xi},
$$

where $\lambda$ is London's penetration depth, $\xi$ the $x y$ coherence length, $\kappa=\sqrt{\frac{m}{M}}$ the anisotropy ratio, $\rho_{N}$ the normal state resistivity, and $\Phi_{o}=\frac{h c}{2 e}$ the flux quantum.

For YBCO films, we can take the typical values $d \simeq$ $12 \stackrel{\circ}{A}, \lambda \simeq 1400 \stackrel{\circ}{A}, \xi \simeq 15 \stackrel{\circ}{A}, \kappa \simeq \frac{1}{5}$ and $\rho_{N} \simeq 100 \mu \Omega \cdot \mathrm{cm}$, yielding

$$
\eta \simeq 3.010^{-6} \frac{\mathrm{Erg} \cdot \mathrm{s}}{\mathrm{cm}^{3}}, \epsilon \simeq 3.410^{-7} \frac{\mathrm{Erg}}{\mathrm{cm}} .
$$

In order to get contact with realizable temperatures in the framework of superconductivity, we define the $\mu$ independent temperature

$$
T_{s}=N T_{o}=\frac{\pi}{2 N} \frac{\hbar}{k_{B}} \frac{\epsilon}{\eta d^{2}} \simeq \frac{10^{2}}{N} K .
$$

For reasonable $N$, ranging from $10^{2}$ to $10^{4}$ and corresponding to a thickness $L$ of the sample between $0.1 \mu \mathrm{m}$ and $10 \mu \mathrm{m}, T_{s}$ can go from $10 \mathrm{mK}$ to $1 \mathrm{~K}$, indeed representing the accessible temperature scale for the problem. In the following, we will assume $T \ll \frac{1}{2 \pi} \frac{\hbar}{k_{B}} \frac{\eta}{\mu}$ in order to satisfy the condition $\frac{\gamma}{\omega} \gg 1$ which has been used in deriving the behavior in the overdamped case. Using the estimate given at the end of this section for $\mu$, this means temperatures below $10^{5} \mathrm{~K}$, and does not constitute any restriction.

In terms of $T_{s}$, the reduced temperature is given by

$$
t=\pi \frac{\sqrt{\epsilon \mu}}{\eta d} \frac{T}{T_{s}} .
$$

Observe that since $T_{s}$ is known but $\mu$ is not, the temperature scale entering $t$ is ambiguous. We are now able to look closer to the order of magnitude of the transition temperatures in the two regimes we have studied, taking as reasonable temperature scale $T_{s}\left(T_{o}=\frac{T_{s}}{N}\right.$ is thus small).

In the underdamped case, $\alpha=\frac{N}{\pi} \frac{\eta d}{\sqrt{\epsilon \mu}} \ll N$, the important reduced temperatures where found to be $t_{A}=1$ and $t_{B}=N$, corresponding to the temperatures $T_{A}=$ $\frac{\alpha}{N} T_{s} \ll T_{s}$ and $T_{B}=\alpha T_{s} \sim T_{s}$. Thus, we conclude that the relevant regimes for the specific heat are in this case the first and second of Eq. (34), that is

$$
C_{v o r} \simeq\left\{\begin{array}{l}
\frac{N}{3}\left(\frac{1}{\alpha^{2}}+\frac{\pi^{2}}{6}\right) \frac{T}{T_{s}}, T \ll \frac{T_{s}}{N} \\
\frac{\pi}{3} \frac{N}{\alpha} \frac{T}{T_{s}}, T \gg \frac{T_{s}}{N}
\end{array} .\right.
$$

In the overdamped case, $\alpha=\frac{N}{\pi} \frac{\eta d}{\sqrt{\epsilon \mu}} \gg N$, we had instead $t_{A}=\frac{1}{\alpha}, t_{B}=\frac{N^{2}}{\alpha}$ and $t_{C}=\alpha$, corresponding to the temperatures $T_{A}=\frac{1}{N} T_{s} \ll T_{s}, T_{B}=N T_{s} \gg T_{s}$ and $T_{C}=\frac{\alpha^{2}}{N} T_{s} \gg T_{s}$. Thus, the relevant regimes for this case are the first and second of Eq. (37), that is

$$
C_{v o r} \simeq\left\{\begin{array}{l}
\frac{N \pi^{2}}{18} \frac{T}{T_{s}}, T \ll \frac{T_{s}}{N} \\
0.98 \sqrt{N \frac{T}{T_{s}}}, T \gg \frac{T_{s}}{N} .
\end{array} .\right.
$$

Multiplying the results (42) and (43) by $k_{B}$ and the density of vortices $\frac{B}{\Phi_{o} L}$ in order to obtain the true specific heat per unit volume, and expliciting all the variables, our final result can be written as 


$$
\begin{aligned}
& \left\{\begin{array}{l}
\frac{\pi}{9} \frac{B}{\Phi_{o}} \frac{k_{B}^{2}}{\hbar}\left(\frac{\eta L}{\epsilon}+\frac{6 \mu}{\eta L}\right) T, T \ll T_{o} \\
\frac{2 \pi}{3} \frac{B}{\Phi_{o}} \frac{k_{B}^{2}}{\hbar} \sqrt{\frac{\mu}{\epsilon}} T, T \gg T_{o}
\end{array}, I \gg 1\right. \\
& \left\{\begin{array}{l}
\frac{\pi}{9} \frac{B}{\Phi_{o}} \frac{k_{B}^{2}}{\hbar} \frac{\eta L}{\epsilon} T, T \ll T_{o} \\
0.98 \sqrt{\frac{8}{\pi}} \frac{B}{\Phi_{o}} \frac{k_{B}^{\frac{3}{2}}}{\hbar^{\frac{1}{2}}} \sqrt{\frac{\eta}{\epsilon}} \sqrt{T}, T \gg T_{o}
\end{array}\right.
\end{aligned}
$$

with

$$
T_{o}=\frac{\pi}{2} \frac{\hbar}{k_{B}} \frac{\epsilon}{\eta L^{2}}, I=\frac{\sqrt{\epsilon \mu}}{\eta d} .
$$

In a previous work by Blatter and Ivlevd the low temperature regime for small magnetic fields was considered using the usual continuum description; this corresponds to the limit $N \rightarrow \infty$ in our description and temperatures $T \ll T_{o}$. In the anisotropic and elastic limit, Eq. (33) of that work for the specific heat reduces to

$$
C_{\text {vor }} \simeq \frac{2}{3} \frac{B}{\Phi_{o}} \frac{k_{B}^{2}}{\hbar} \frac{\eta}{\epsilon} \int_{k_{z \min }}^{\infty} \frac{d k_{z}}{k_{z}^{2}} .
$$

Our finite $N$ case can be recovered with the identifications $k_{z} \rightarrow \nu n, d k_{z} \rightarrow \nu\left(\nu=\frac{\pi}{L}\right)$, meaning $k_{z \min }=\frac{\pi}{L}$ and

$$
\int_{k_{z \min }}^{\infty} \frac{d k_{z}}{k_{z}^{2}} \rightarrow \frac{1}{\nu} \sum_{n=1}^{N-1} \frac{1}{n^{2}} \simeq \frac{\pi}{6} L .
$$

In this way, one matches the linear and $\mu$-independent results of the first and third row of (44), which are relevant for $T \ll T_{o}$ and coincide in the continuum limit on wich we are focusing.

Note that out result Eq. (43), case $T \gg \frac{T_{s}}{N}$, would be modified by the introduction of a lower cut-off $k_{z \min }$ in the following way

$$
C_{\text {vor }} \simeq 2 \sqrt{N \frac{T}{T_{s}}} \int_{\sqrt{N \frac{T}{T_{s}} \frac{d}{\pi} k_{z m i n}}}^{\sqrt{N \frac{T}{T_{s}}}} d z z^{4} \tilde{\Psi}^{\prime}\left(z^{2}\right) .
$$

We took $k_{z \text { min }}=\frac{\pi}{L}$, but the behavior $C_{v o r} \sim \sqrt{N \frac{T}{T_{s}}}$ would hold also for other possible $k_{z \min }$, provided $k_{z \min } \ll \frac{\pi}{d} \sqrt{\frac{T_{s}}{N T}}$.

The behavior for large magnetic fields has been studied by Bulaevskii and Maleyl. In this case, the specific heat is still linear in the temperature, but has a different dependence on the magnetic field and the microscopic parameters of the vortices, and the result can not be expressed as a function of only $\mu, \eta$ and $\epsilon$; this signals that in this regime the vortices do not retain their line structure and other kind of configuration become important.
Summarizing, for $T \ll T_{o}$, and in the continuum limit $N \rightarrow \infty$, we find the universal behavior already studied in the litterature 7 . For $T \gg T_{o}$ and in the whole range of superconductivity temperatures we find instead a different behavior depending on the value of the parameter $I$. In the underdamped case $I \gg 1$ the specific heat is linear in the temperature and depends only on inertia and not on friction, whereas in the overdamped case $I \ll 1$ it goes like the square root of the temperature and depends only on friction and not on inertia.

It is important to observe that for $N$ ranging from $10^{2}$ to $10^{4}$, the temperature $T_{o}$ is very small and varies from $1 \mu K$ to $10 \mathrm{mK}$. This suggest that an interesting experimentally observable regime should be $T>T_{o}$.

As an example of theoretical estimate, one can approximate the mass density $\mu$ with its electronic and electer magnetic contributions. These are found found to bet. 13

$$
\mu_{e l}=\frac{2}{\pi^{3}} m_{e} k_{F}, \mu_{e m}=\left(\frac{\Phi_{o}}{4 \pi c \xi}\right)^{2} .
$$

For YBCO films, the Fermi momentum is $k_{F}=$ $0.5 A^{-1}$, and one obtains $\mu_{e l} \simeq 2.910^{-21} \frac{\mathrm{gr}}{\mathrm{cm}}, \mu_{e m} \simeq$ $1.210^{-22} \frac{\mathrm{gr}}{\mathrm{cm}}$. This would mean $I \simeq 0.1$, which does not corresponds clearly to any of the two damping regimes. Thus, if one uses this theoretical estimate, the thermodynamics should lie somewhere inbetween the underdamped and overdamped regime that we have considered in more detail. However, trusting other conventional arguments $\mathbf{B}$, the overdamped case should be the relevant one. An experimental measure of the specific heat for $T \gg T_{o}$ could thus provide specific informations on this important issue.

\section{EFFECT OF THE MAGNUS FORCE}

In this section we will discuss the influence of a possible Magnus force on the thermodynamics and show how our results for the specific heat generalize to the case in which both the Magnus effect and friction are important.

The Magnus force corresponds to a term

$$
\delta{ }^{*} \dot{\mathbf{q}}
$$

in the equation of motion (11) of the vortex line, where ${ }^{*} \mathbf{q}=\mathbf{q} \times \mathbf{z}$ is the dual of the $x y$ position. This term can be accounted for in the thermodynamics by adding in the Euclidean effective action (3) the term

$$
\int_{0}^{L} d z \int_{0}^{\beta} d \tau \frac{i}{2} \delta \mathbf{q}^{*} \dot{\mathbf{q}} .
$$

Actually, the Magnus force is rather controvertial 14 . In the low magnetic field limit, by estimating $\delta$ from the number density of the superconducting fluid, one would get a value of the same order of $\eta$ as in eq. (39). 
Accordingly, the partition function for each of the modes of the vortex line engoes the following modification

$$
\begin{aligned}
\prod_{k=1}^{+\infty}\left[1+\frac{\gamma}{\omega} \frac{1}{k}+\left(\frac{\Omega_{n}}{\omega}\right)^{2} \frac{1}{k^{2}}\right]^{-2} \rightarrow \\
\rightarrow \prod_{k=1}^{+\infty}\left\{\left[1+\frac{\eta}{\mu \omega} \frac{1}{k}+\left(\frac{\Omega_{n}}{\omega}\right)^{2} \frac{1}{k^{2}}\right]^{2}+\left(\frac{\delta}{\mu \omega}\right)^{2} \frac{1}{k^{2}}\right\}^{-1} \\
\quad=\prod_{k=1}^{+\infty}\left|1+\frac{\hat{\gamma}}{\omega} \frac{1}{k}+\left(\frac{\Omega_{n}}{\omega}\right)^{2} \frac{1}{k^{2}}\right|^{-2}
\end{aligned}
$$

We have introduced the complex damping frequency

$$
\hat{\gamma}=\frac{\eta+i \delta}{\mu} .
$$

This leads to the following modifications in the expressions for the partition functions

$$
\begin{aligned}
& \tilde{\Gamma}^{2}\left(\frac{\gamma}{\omega}\right) \rightarrow \tilde{\Gamma}\left(\frac{\hat{\gamma}}{\omega}\right) \tilde{\Gamma}\left(\frac{\hat{\gamma}^{*}}{\omega}\right) \\
& \tilde{\Gamma}^{2}\left(\frac{\frac{\gamma}{2} \pm i \xi_{n}}{\omega}\right) \rightarrow \tilde{\Gamma}\left(\frac{\frac{\hat{\gamma}}{2} \pm i \hat{\xi}_{n}}{\omega}\right) \tilde{\Gamma}\left(\frac{\hat{\gamma}^{*} \pm i \hat{\xi}_{n}^{*}}{\omega}\right)
\end{aligned}
$$

where

$$
\hat{\xi}_{n}=\sqrt{\Omega_{n}^{2}-\frac{\hat{\gamma}^{2}}{4}}, \quad \hat{\xi}_{n}^{*}=\sqrt{\Omega_{n}^{2}-\frac{\hat{\gamma}^{* 2}}{4}} .
$$

All the analysis done for the dissipative case goes through without any major difficulty. Underdamped and overdamped cases now means with respect to the modulus $\eta_{e}$ of the complex friction coefficient $\hat{\eta}=\eta_{e} e^{i \phi}$ for which

$$
\eta_{e}=\sqrt{\eta^{2}+\delta^{2}}, \phi=\arctan \frac{\delta}{\eta} .
$$

The result (44) generalizes to

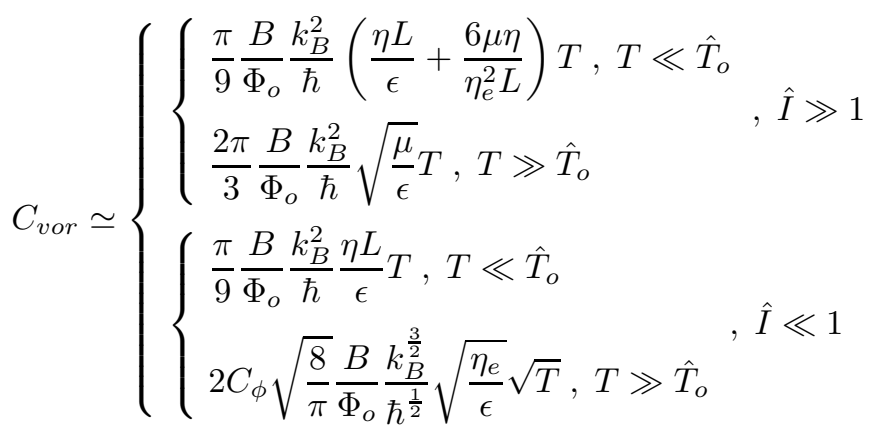

with

$$
\hat{T}_{o}=\frac{\pi}{2} \frac{\hbar}{k_{B}} \frac{\epsilon}{\eta_{e} L^{2}}, \hat{I}=\frac{\sqrt{\epsilon \mu}}{\eta_{e} d} .
$$

The constant $C_{\phi}$ appearing in the overdamped case now depends on the phase $\phi$ and is again given by an integral

$$
C_{\phi}=\Re\left\{e^{-2 i \phi} \int_{0}^{+\infty} d z z^{4} \tilde{\Psi}^{\prime}\left(e^{-i \phi} z^{2}\right)\right\} .
$$

Since the relevant asymptotic behavior of the integrand for $z \rightarrow 0$ is independent of $\phi$, we expect $C_{\phi}$ to depend only weakly on it. In fact, the to extreme values corresponding to $\eta \neq 0, \delta=0(\phi=0)$ and $\eta=0, \delta \neq 0$ $\left(\phi=\frac{\pi}{2}\right)$ are given by the integrals (A11) and A12 of appendix A,

$$
C_{o}=0.490, C_{\frac{\pi}{2}}=0.346 .
$$

For arbitrary $\phi, C_{\phi}$ is of the same order of magnitude and can be computed numerically.

Observe finally that in the overdamped case, in the sense explained above (with respect to $\eta_{e}$ ), the specific heat depends on the square root of the temperature and on the effective damping coefficient $\eta_{e}=\sqrt{\eta^{2}+\delta^{2}}$. In the ultra clean limit $\left(\eta_{e}=\delta\right.$ ) this was already noticed bye Blatter and Ivlevt, who also found $C_{v} \sim \sqrt{T}$ in this case.

Aknowledgements. This work has been partially supported by EEC contract ERBFMRXCT 96-0045.

\section{APPENDIX A: PROPERTIES OF T-FUNCTIONS}

In this appendix, me recall some formulae involving $\Gamma$ functions and other 15 . First of all, there are the infinite product representations of the hyperbolic sine and the $\Gamma$-function, given respectively by

$$
\begin{aligned}
& \sinh z=z \prod_{k=1}^{+\infty}\left[1+\left(\frac{z}{\pi k}\right)^{2}\right], \\
& \Gamma(z)=\lim _{N \rightarrow+\infty} \frac{N^{z}}{z} \prod_{k=1}^{N}\left(1+\frac{z}{k}\right)^{-1} .
\end{aligned}
$$

We recall the two useful formulae, holding away from the real negative axis,

$$
\begin{aligned}
& \Gamma(z) \underset{|z| \rightarrow+\infty}{\longrightarrow} \sqrt{2 \pi} z^{z-\frac{1}{2}} e^{-z}\left(1+\frac{1}{12 z}+\ldots\right), \\
& \Gamma(z+x) \underset{|z| \rightarrow+\infty}{\longrightarrow} z^{x} \Gamma(z) .
\end{aligned}
$$

Notice the simplified asymptotic behaviors of the functions defined in Eqs. (10) and 29

$$
\begin{aligned}
& \tilde{\Gamma}(z) \underset{|z| \rightarrow+\infty}{\longrightarrow} 1+\frac{1}{12 z}+\ldots \simeq 1, \\
& \tilde{\Psi}^{\prime}(z) \underset{|z| \rightarrow+\infty}{\longrightarrow} \frac{1}{6 z^{3}}+\ldots \simeq 0 .
\end{aligned}
$$

All the behavior for small arguments can be deduced from the one of the $\Gamma$-function which has a simple pole in the origin. In particular 


$$
\begin{aligned}
& \tilde{\Gamma}(z) \underset{|z| \rightarrow 0}{\longrightarrow} \frac{1}{\sqrt{2 \pi z}}, \\
& \tilde{\Psi}^{\prime}(z) \underset{|z| \rightarrow 0}{\longrightarrow} \frac{1}{2 z^{2}} .
\end{aligned}
$$

The $\tilde{\Gamma}$ and $\tilde{\Psi}$ functions are related to hyperbolic functions at immaginary arguments

$$
\begin{aligned}
& \tilde{\Gamma}(i x) \tilde{\Gamma}(-i x)=\frac{e^{\pi x}}{2 \sinh \pi x} \\
& \tilde{\Psi}^{\prime}(i x)+\tilde{\Psi}^{\prime}(-i x)=-\left(\frac{\pi}{\sinh \pi x}\right)^{2}
\end{aligned}
$$

Finally, we will need the following integrals

$$
\begin{aligned}
& C_{o}=\int_{0}^{+\infty} d z z^{4} \tilde{\Psi}^{\prime}\left(z^{2}\right)=0.490 \\
& C_{\frac{\pi}{2}}=\frac{1}{2} \int_{0}^{+\infty} d z\left(\frac{\pi z^{2}}{\sinh \pi z^{2}}\right)^{2}=0.346 .
\end{aligned}
$$

\section{APPENDIX B: LATTICE REGULARIZATION}

We present here some results about divergent quanti-

ties computed with the lattice regularization. Using the lattice spectrum

$$
\Omega_{n}=\frac{2 N}{\pi} \sin \left(\frac{\pi}{2} \frac{n}{N}\right) \Omega
$$

and the formulae

$$
\begin{aligned}
& \sum_{n=1}^{N-1} 2 \sin \left(\frac{\pi}{2} \frac{n}{N}\right)=\cot \frac{\pi}{4 N}-1 \simeq \frac{4 N}{\pi}-1-\frac{\pi}{12 N} \\
& \prod_{n=1}^{N-1} 2 \sin \left(\frac{\pi}{2} \frac{n}{N}\right)=\sqrt{N} \\
& \sum_{n=1}^{N-1} \frac{\frac{\pi}{2 N}}{\sin \left(\frac{\pi}{2} \frac{n}{N}\right)} \simeq \ln G N, G=2.267
\end{aligned}
$$

it easily follows that

$$
\begin{aligned}
& \sum_{n=1}^{N-1} \Omega_{n} \simeq\left[\frac{N}{\pi}\left(\frac{4 N}{\pi}-1\right)-\frac{1}{12}\right] \Omega, \\
& \prod_{n=1}^{N-1} \Omega_{n}=\left(\frac{N}{\pi} \Omega\right)^{N-1} \sqrt{N}, \\
& \sum_{n=1}^{N-1} \frac{1}{\Omega_{n}} \simeq \frac{1}{\Omega} \ln G N .
\end{aligned}
$$

${ }^{1}$ For a recent review, see G. Blatter, M. V. Feigel'man, V. B. Geshkenbein, A. I. Larkin and V. M. Vinokur, Rev. Mod. Phys. 66, 1125 (1994).

${ }^{2}$ G. Blatter, V. B. Geshkenbein and V. M. Vinokur, Phys. Rev. Lett. 66, 3297 (1991).

${ }^{3}$ B. I. Ivlev, Y. N. Ovchinnifov and R. S. Thompson, Phys. Rev. B 44, 7023 (1991).

${ }^{4}$ G. Blatter and V. M. Vinokur, Phys. Rev. B 47, 2725 (1993).

${ }^{5}$ L. N. Bulaevskii and M. P. Maley, Phys. Rev. Lett. 71, 3541 (1993).

${ }^{6}$ L. M. Bulaevskii, J. H. Cho, M. P. Maley, P. Kes, Q. L Li, M. Suenaga and M. Ledvij, Phys. Rev. B 50, 3507 (1994).

${ }^{7}$ G. Blatter, B. I. Ivlev, Phys. Rev. B 50, 10272 (1994).

${ }^{8}$ M. J. Stephen, Phys. Rev. Lett. 72, 1534 (1994).

${ }^{9}$ A. O. Caldeira and A. J. Leggett, Ann. Phys. 149, 374 (1983).

${ }^{10}$ U. Weiss, Ser. Mod. Cond. Mat. Phys. 2, "Quantum Dissipative Systems", World Scientific (1993).

${ }^{11}$ U. Weiss, Z. Phys. B 55, 87 (1984).

12 J. Bardeen and M. J. Stephen, Phys. Rev. 140, A1197 (1965).

${ }^{13}$ H. Suhl, Phys. Rev. Lett. 14, 226 (1965).

${ }^{14}$ For a recent survey see D. J. Thouless, P. Ao, Q. Niu, M. R. Geller and C. Wexler, "Quantized Vortices in Superfluids and Superconductors", cond-math/9709127, and references therein.

${ }^{15}$ I. S. Gradshteyn and I. M. Ryzhik, "Table of Integrals, Series, and Products", Accademic Press (1980). 